\title{
Laparoscopic-assisted Transanal Total Mesorectal Excision for Middle-Low Rectal Carcinoma: A Clinical Study of 19 Cases
}

\author{
HONG-WEI YAO ${ }^{1}$, GUO-CONG WU ${ }^{1}$, YING-CHI YANG ${ }^{1}$, LAN JIN ${ }^{1}$, \\ ZHI-PENG ZHANG ${ }^{2}$, NING CHEN $^{2}$ and ZHONG-TAO ZHANG ${ }^{1}$ \\ ${ }^{1}$ Department of General Surgery, Beijing Key Laboratory of Cancer Invasion and Metastasis Research \\ and National Clinical Research Center for Digestive Diseases, Beijing Friendship Hospital, \\ Capital Medical University, Beijing, P.R. China; \\ ${ }^{2}$ Department of General Surgery, Peking University Third Hospital, Beijing, P.R. China
}

\begin{abstract}
Aim: To explore the safety, feasibility and shortterm outcomes of laparoscopic-assisted transanal total mesorectal excision (La-TaTME) of middle-low rectal carcinoma. Materials and Methods: This retrospective research collected and analyzed the clinical data of 19 patients diagnosed with middle and low rectal carcinoma who underwent La-TaTME from August 2015 to February 2017. Results: No case was converted to laparotomy. In none of the enrolled cases, did the circumferential resection margin test positive by histopathology examination. Also, there was no detected residual tumor at the proximal and distal resection margins. The rate of postoperative morbidity was $15.8 \%$ (Clavien-Dindo grade 2). During the follow-up period, no local recurrence or metastasis was observed. Conclusion: La-TaTME is a safe alternative to standard laparoscopic TME in middle-low rectal carcinoma when operated by an experienced colorectal surgeon. However, large-scaled randomized multi-centered comparative trials are still needed to further test its oncological effectiveness.
\end{abstract}

Total mesorectal excision (TME) is the most effective surgical way to treat patients with middle-low rectal carcinoma (1). However, incomplete surgical resection of the mesorectum can increase the risks of local recurrence (2). For patients with middle-low rectal cancer who have concurrent obesity, are male, have huge-sized tumors, narrow pelvis and

Correspondence to: Zhong-tao Zhang, MD, Ph.D., Department of General Surgery, Beijing Key Laboratory of Cancer Invasion and Metastasis Research and National Clinical Research Center for Digestive Diseases, Beijing Friendship Hospital, Capital Medical University, No.95 Yong-an Road, Xi-cheng District, Beijing 100050, P.R. China. Tel: +86 1063138768, e-mail: zhangzht@medmail.com.cn

Key Words: Middle-low rectal cancer, total mesorectal excision, transanal total mesorectal excision. received neoadjuvant chemoradiotherapy, it is very difficult to perform TME. Compared to transabdominal TME, transanal TME (TaTME) and laparoscopic-assisted transanal TME (La-TaTME) have the minimally-invasive advantages of natural orifice transluminal endoscopic surgery (NOTES) and of no abdominal incisions $(3,4)$. This study reviewed and analyzed the clinical data of patients who underwent LaTaTME by a surgical team from August 2015 to February 2017. The study aimed to explore the safety, feasibility and short-term outcomes of La-TaTME in the treatment of patients with middle-low rectal carcinoma.

\section{Materials and Methods}

Patients and clinical data. All the patients were enrolled from the Department of Surgery of Beijing Friendship Hospital of Capital Medical University, Beijing, China, and confirmed as suffering from colorectal cancer diagnosed through colonoscopy and histopatho-logical examination. All these patients had middle-low rectal cancer (measured by magnetic resonance imaging (MRI) defined as the distance from the lower margin of the tumor to the anal verge being between 2 and 10 $\mathrm{cm})$. Before the operation, transanal ultrasonography and MRI of the rectum were conducted in order to establish the tumor/node $(\mathrm{T} / \mathrm{N})$ staging. Abdominal and chest computed tomography (CT) was also performed to exclude unresectable distant metastases. The clinical cancer staging of all enrolled cases was not beyond $\mathrm{cT}_{3} \mathrm{~N}_{1} \mathrm{M}_{0}$ and classified into the American Society of Anesthesiologists (ASA) grades I-III. The specific data of the 19 cases are shown in Table I.

Surgical procedures. The operations of all enrolled patients were performed by the same team of surgeons. After the patients were given general anesthesia, tracheal intubation was performed and the patients placed in lithotomy position. The abdominal phase was performed before the transanal phase of the operation.

Transabdominal surgery. At the upper margin of the umbilicus, one $10-\mathrm{mm}$ trocar was inserted to establish the pneumoperitoneum and the laparoscope was also inserted as the observation port. During the transabdominal surgery, a traction belt was inserted through the upper mesorectum in order to occlude the rectal lumen. There were two advantages to using the traction belt; 
firstly, it could be used to pull the rectum to expose the operation space during the pelvic operation and, secondly, it could be used to block the rectum to avoid the serious flatulence when insufflated with $\mathrm{CO}_{2}$ during the transanal phase of the operation. High ligation of the inferior mesenteric artery, lymph node dissection and skeletalization of the intestinal wall at the level of the anastomosis were all performed. Using the laparoscope, following the same principles of TME, the mesorectum was mobilized to the pelvic floor around the levels of the 4th $\sim$ th sacral vertebrae and of peritoneal reflection.

Transanal surgery. After disinfection of the perineal region, diluted iodophor solution was injected through the anus to irrigate the rectum. One single-port laparoscopic instrument was inserted through the anus (Triport ${ }^{\mathrm{TM}}$; Olympus, Tokyo, Japan) or SILS ${ }^{\mathrm{TM}}$ (Medtronic, Minneapolis, MN, USA) or the transanal endoscopic microsurgery (TEM) operation system was inserted. The rectum was insufflated to a pressure of $10 \mathrm{~mm} \mathrm{Hg}$. At the distance of 1-5 $\mathrm{cm}$ from the lower verge of the tumor, the rectum was stitched and occluded. Diluted iodine solution was used again to irrigate the rectum to prevent the tumor cells and bacteria from being polluted. Only $0.5 \mathrm{~cm}$ from the far end of the suture, electrocautery was used to cut through the rectal wall into the pelvic floor. Firstly, the distal mesorectum was mobilized in a "down to up" manner in the TME anatomic plane, until it reached the mobilization location of the transabdominal surgery. Special attention needed to be paid to the angle between the rectum and the anal canal to avoid damage to the pre-sacral veins during dissection of the postrectal space (Figure 1).

After dissection of the postrectal space, the dissection of the prerectal space was conducted and mobilization was performed in a "bottom-up" manner until it reached peritoneal reflextion, where the transabdominal surgery mobilization was carried out. In the case of male patients, due attention was needed to avoid damage to the prostate and the seminal vesicles and, in the case of female patients, (Figure 2) caution was needed to avoid damage to the vagina. One of the surgical difficulties lies in the lateral mobilization of the mesorectum where the blood veins and nerves could be easily damaged. We feel that it is easier to conduct lateral mobilization after the dorsal and ventral mobilization were carried out. With the endoscope, our research team applied the harmonic scalpel or electronic hook to conduct sharp dissection so that the correct anatomy layer could be identified. Total mesorectal excision (TME) was completed when it reached the level of the abdominal operations. The TME specimen was taken out through the anus.

Anastomosis or colostomy. In those cases that underwent anastomosis, the circular stapler was used. The anvil of the circular stapler was inserted through the cut end of the sigmoid colon, which was then placed into the pelvic cavity through the anus. Transanal purse-string suture was performed to close the distal rectal stump and the central pole of the circular stapler was inserted through the anus. After reconstitution of the pneumoperitoneum, with the aid of the laparoscope, the sigmoid colon and the rectum were anastomosed with the adoption of the circular stapler. In the cases where the stump of the rectum was very close to the dental line (around 0-1 cm), the circular stapler was not able to be used for anastomosis but rather a colon-anal anastomosis approach was adopted. This approach took the cut end of the sigmoid colon out
Table I. Clinical data of the 19 patients enrolled in the study.

\begin{tabular}{|c|c|}
\hline Variable & \\
\hline Age in years (range) & $65(32-82)$ \\
\hline \multicolumn{2}{|l|}{ Gender, $\mathrm{n}(\%)$} \\
\hline Male & $15(78.9 \%)$ \\
\hline Female & $4(21.1 \%)$ \\
\hline BMI $\left(\mathrm{kg} / \mathrm{m}^{2}\right)$ & $22.7(16.3-28.2)$ \\
\hline \multicolumn{2}{|l|}{ Distance from tumor's lower } \\
\hline margin to anal verge $(\mathrm{cm})$ & $4.5(2-10)$ \\
\hline \multicolumn{2}{|l|}{ cTNM staging-T, n $(\%)$} \\
\hline $\mathrm{cT}_{1}$ & $3(15.8 \%)$ \\
\hline $\mathrm{cT}_{2}$ & $6(31.6 \%)$ \\
\hline $\mathrm{cT}_{3}$ & $10(52.6 \%)$ \\
\hline $\mathrm{cT}_{4}$ & $0(0 \%)$ \\
\hline \multicolumn{2}{|l|}{ cTNM staging-N, n (\%) } \\
\hline $\mathrm{cN}_{0}$ & $11(57.9 \%)$ \\
\hline $\mathrm{cN}_{1}$ & $7(36.8 \%)$ \\
\hline $\mathrm{cN}_{2}$ & $1(5.3 \%)$ \\
\hline \multicolumn{2}{|l|}{ ASA grading, $\mathrm{n}(\%)$} \\
\hline I & $6(31.6 \%)$ \\
\hline II & $10(52.6 \%)$ \\
\hline III & $3(15.8 \%)$ \\
\hline \multicolumn{2}{|c|}{ Neoadjuvant chemoradiotherapy, n (\%) } \\
\hline No & $14(73.7 \%)$ \\
\hline Yes & $5(26.3 \%)$ \\
\hline
\end{tabular}

BMI, Body mass index; TNM, tumor-node-metastasis; ASA, American Society of Anesthesiologists.

through the anal verge, fully exposed the anal canal or the short stump of the rectum and performed the colon-anal or colo-rectal anastomosis by interrupted full-thickness suture. Based on the patient's condition, the distance between the anastomosis and the anal edge, as well as the quality of the anastomosis, the surgical team would then decide whether to perform the ileostomy as a protective measure. For those patients in need, the 13-mm trocar site in the right lower abdomen was expanded to mobilize the terminal ileum for ileostomy. A drainage tube was inserted into the pelvis behind the anastomosis. For those patients whose original surgical design was to perform a permanent colostomy, transanal suture was conducted to close the short stump of the rectum, while the trocar site on the lower left abdomen was expanded to perform the sigmoid colostomy.

In two of the cases, the patients also suffered from synchronous single liver metastasis. In those cases, the liver metastasis was firstly resected by hepatic surgeons through laparoscopic surgery. Subsequently, colorectal surgeons conducted La-TaTME, taking out the liver and the rectal specimens through the anus. In another case, the patient suffered from both low rectal cancer and prostate cancer. After colorectal surgeons performed La-TaTME, an ultrasound doctor worked with urologist surgeons to insert radioactive Iodine125 seeds through the anus.

Follow-ups. Those patients with stage III or IV rectal cancer underwent postoperative chemotherapy. After the operations, patients had follow-up checks every three months. The follow-up checks were completed by April 2017. 


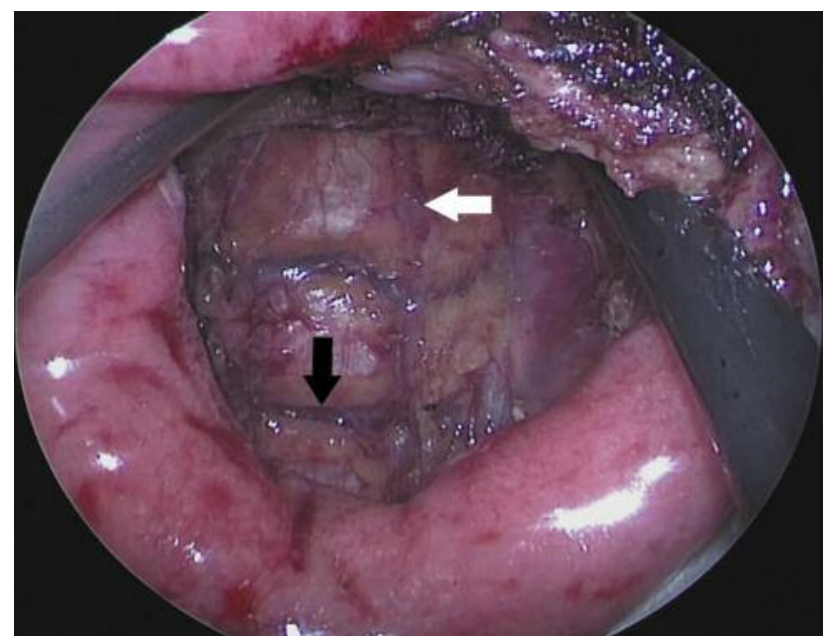

Figure 1. Transanal view of middle sacral vein as shown by the white arrow and sacral transverse vein as shown by the black arrow.

\section{Results}

Clinicopathological characteristics of the enrolled patients. The perioperative clinicopathological data of 19 patients are presented in Table II. In none of the enrolled cases, was the circumferential resection margin tested positive by the histopathology examination. In addition, there was no detected tumor remaining at the proximal and distal resection margins. The median length of the specimen was $18 \mathrm{~cm}$ (range $=12-24 \mathrm{~cm}$ ) and the median number of the harvested lymph nodes was 16 (range=6-32).

In one of the cases there was an intraoperative complication. After transanal TME, the cut end of the sigmoid colon was found to be ischemic. Once the splenic flexure of colon was mobilized and a 5-cm-long section of sigmoid colon was resected, the colo-rectal end-to-end anastomosis was performed. None of the cases in the cohort was converted to open surgery. The median preoperative blood loss was $50 \mathrm{ml}$ (range $=30-100 \mathrm{ml}$ ). The median operation time was $290 \mathrm{~min}$ (range $=190-420 \mathrm{~min}$ ). There was no case of leakage at the anastomosis.

The median time of postoperative oral intake was 2 days (range=1-9) and the median duration of postoperative hospitalization was 7 days (range=5-21). In this cohort, there were 3 cases suffering from postoperative complications (Clavien-Dindo grade 2). One case was of presacral infection, which was relieved after drainage and antibiotic treatment. The other two cases with complications were of incomplete intestinal obstruction; they were relieved by gastrointestinal decompression and parenteral nutrition.

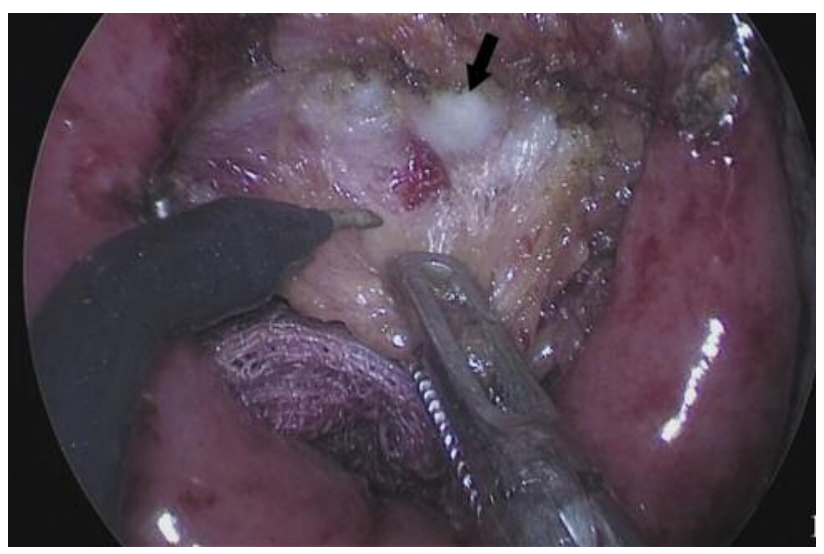

Figure 2. Dissection of the anterior fascia propria of mesorectum, with the black arrow showing the seminal vesicle.

Follow-up. All the patients had follow-up visits and the median follow-up duration was 12 (range=2-20) months. There was no case of local recurrence or distal metastasis. There was no case of death.

\section{Discussion}

TME has been shown to improve the prognosis of rectal cancer patients (5). Although many randomized comparative trials have demonstrated the surgical effects and the shortterm outcomes of laparoscopic TME surgery $(6,7)$, local recurrence rates and long-term survival are less wellreported. Recent clinical trials, such as COREAN and COLOR II, have shown that laparoscopic surgery in patients with rectal cancer is associated with rates of local recurrence and disease-free and overall survival similar to those for open surgery $(8,9)$. Nevertheless, the research results of ACOSOG Z6051 and ALacaRT have shown that the use of laparoscopic resection, compared with open resection, fail to meet the criterion for non-inferiority for pathologic outcomes $(10,11)$. Regarding clinical oncologic outcomes, the findings do not support the use of laparoscopic resection in these patients with stage II or III rectal cancer. Therefore, the quality of the TME specimen of mid-low rectal cancer is still the key question regarding whether laparoscopic TME is the "golden standard" surgery. In cases of mid-low rectal cancer, the greatest difficulty lies in the complete resection of the distal mesorectum, especially for obese males or patients with a narrow pelvis (12). If the traditional approach is taken, no matter whether open or laparoscopic surgery is adopted, there are difficulties with exposure of pelvic space, 
Table II. Perioperative clinicopatholigical characteristics of the 19 patients enrolled in the study.

\begin{tabular}{|c|c|}
\hline Variable & \\
\hline \multicolumn{2}{|l|}{ Surgical procedures, n (\%) } \\
\hline LAR and colo-rectal anastomosis & $12(63.2 \%)$ \\
\hline LAR+ISR and colo-anal anastomosis & $4(21.1 \%)$ \\
\hline LAR and permanent colostomy & $3(15.8 \%)$ \\
\hline Operation time (min) & $290(190-420)$ \\
\hline Intraoperative blood loss (ml) & $50(30-100)$ \\
\hline Postoperative return to normal activities (days) & $2(1-3)$ \\
\hline Postoperative recovery of oral intake (days) & $2(1-9)$ \\
\hline \multicolumn{2}{|l|}{ pTNM staging-T, $\mathrm{n}(\%)$} \\
\hline $\mathrm{pT}_{0}$ & $2(10.5 \%)$ \\
\hline $\mathrm{pT}_{1}$ & $2(10.5 \%)$ \\
\hline $\mathrm{pT}_{2}$ & $5(26.3 \%)$ \\
\hline $\mathrm{pT}_{3}$ & $10(52.6 \%)$ \\
\hline $\mathrm{pT}_{4}$ & $0(0 \%)$ \\
\hline \multicolumn{2}{|l|}{ pTNM staging-N, n (\%) } \\
\hline $\mathrm{pN}_{0}$ & $14(73.7 \%)$ \\
\hline $\mathrm{pN}_{1}$ & $3(15.8 \%)$ \\
\hline $\mathrm{pN}_{2}$ & $2(10.5 \%)$ \\
\hline Number of harvested lymph nodes & $16(6-32)$ \\
\hline \multicolumn{2}{|l|}{ Degree of tumor differentiation, n (\%) } \\
\hline No tumor cell & $2(10.5 \%)$ \\
\hline Well-differentiated & $4(21.1 \%)$ \\
\hline Moderately differentiated & $7(36.8 \%)$ \\
\hline Poorly differentiated & $6(31.8 \%)$ \\
\hline Size of tumor $(\mathrm{cm})$ & $3.5(0.5-6.8)$ \\
\hline Length of specimen $(\mathrm{cm})$ & $18(12-24)$ \\
\hline \multicolumn{2}{|l|}{ Circumferential resection margin, n (\%) } \\
\hline Positive $(\leq 1 \mathrm{~mm})$ & $0(0 \%)$ \\
\hline Negative (>1 mm) & $19(100 \%)$ \\
\hline \multicolumn{2}{|l|}{ Distal resection margin of the rectum, $\mathrm{n}(\%)$} \\
\hline Positive & $0(0 \%)$ \\
\hline Negative & $19(100 \%)$ \\
\hline
\end{tabular}

LAR, Low anterior resection.

utilization of surgical instruments and protection of the nerves of the pelvic floor (13). Also, the rate of positive circumferential resection margin will increase $(7,14)$.

TaTME, also called "bottom-up" TME surgery, has both the advantages of the NOTES and the TEM. Initial research findings have shown that this surgical approach is both safe and feasible $(12,15)$. Transanal surgery starts from where it is most difficult, which provides a better vision and more surgical space for the complete resection of distal mesorectum. Compared to the transabdominal approach, TaTME has great advantages in dealing with the low rectum and the pelvic floor (16).

Our surgical team has conducted La-TaTME for mid-low rectal cancer patients since August 2015 with 19 cases conducted until February 2017. One of the cases had intraoperative complications. As mentioned above, after transanal TME, the divided end of the sigmoid colon was found to be ischemic. The main reasons were that the length of sigmoid colon was relatively short and the splenic flexure was not mobilized during the transabdominal operation. Therefore, when the specimen was taken out of the anus after TaTME, it was relatively difficult to pull the proximal sigmoid colon via the anus. There was tension from the sigmoid colon mesentery and the mesenteric vessels were compromised causing ischemia of the transected end of the sigmoid colon. After the splenic flexure of colon was mobilized with the help of the laparoscope, it was easier to pull out the proximal sigmoid colon, leading to the resection of an additional 5-cm-long section of sigmoid colon. Finally, the colon-rectal end-to-end anastomosis was performed. The rest of the 18 cases were completed successful.

In two of the cases, the patients also suffered from synchronous single liver metastasis. In those cases, the liver metastasis was firstly resected by hepatic surgeons through laparoscopic surgery and, then, colorectal surgeons conducted La-TaTME and removed the liver and the rectal specimens through the anus. This approach helped to ensure both radical resection and the least surgical trauma. In another case, the patient suffered from both low rectal cancer and prostate cancer. After colorectal surgeons performed the procedure of La-TaTME, an ultrasound doctor worked with urologist surgeons to insert radioactive Iodine-125 seeds through the anus.

There were two cases of postoperative incomplete small intestinal obstruction, whereas, in one of the cases, the patient had a history of open abdominal surgeries and suffered from intraoperative extensive abdominal adhesions. With conservative treatment, both patients recovered. There was one case of postoperative presacral infection in the patient with both rectal cancer and prostate cancer, with the main reason for the complication being the duration of transanal operations, including procedures for both rectum and prostate.

It is worth mentioning that the majority of the enrolled patients were of older age (the median age was 65 with 10 patients over 70 years old). Because of the risks of potential anastomotic leakage and postoperative defecation function, a permanent colostomy was performed in three cases of patients with low or ultra-low rectal cancer in which the trocar site on the lower left abdomen was expanded to perform the sigmoid colostomy. Transanal suture was conducted to close the short stump of the rectum. During the laparoscopic colostomy, we expanded a 5-mm trocar site to $13 \mathrm{~mm}$ on the left lower abdomen, which was used for the insertion of linear stapler and the dissection of the sigmoid colon, and we then further expanded it to $30 \mathrm{~mm}$ for the creation of the colostomy. This helped to minimize trauma.

At the start of the transanal surgery, we could use the scale to accurately measure and record the lower margin of the resection to make sure that the distal margin was safe 
enough, which was important to ensure the radical nature of the surgery and enhance the rate of anal sphincter conservation for patients with low or ultra-low rectal cancer, especially for patients with a low anterior wall tumor. For those patients with low rectal cancer, it is very difficult to preserve the anal sphincter by way of transabdominal open or laparoscopic surgery. Even for male patients with narrow pelvis, this "bottom-up" approach has the advantage of sound surgical vision and reduces the chances of damaging the prostate, seminal vesicles or presacral veins.

In the evaluation of a new surgical approach for rectal cancer, it is, of course, more important to examine the pathological results of the surgical specimens and the longterm oncological outcomes. Among the independent factors affecting the postoperative recurrence rates and the longterm survival, are the completeness of total mesorectal excision and the negative circumferential resection margin of the mesorectum $(17,18)$. The surgical specimens of this cohort demonstrated completeness of mesorectum and rectum. All the patients had follow-up checks and, during the observation period, there was no local recurrence or distal metastases. However, since the median length of follow-up in the study was only 12 months, a longer followup range is needed to evaluate the long-term outcomes of La-TaTME.

A new surgical approach is also faced with new complications. Since the surgical direction of TaTME is opposite from the transabdominal surgery, during the course of mobilizing the distal mesorectum, special attention is needed. There have been reports of damage to the urethra (19). We believe that the operational vision is much better in the case of transanal surgery as compared to transabdominal surgery. With due precautions, damage to organs could be avoided. In the transanal operations, caution is needed to avoid the occurrence of presacral absecess. Before the rectal wall incision is performed transanally, the rectal cavity needs to be irrigated repeatedly. The insertion of the pelvic drainage tube is necessary before the end of the operation. Given that low rectal cancer resection could easily lead to urinary dysfunction, prolonged catheter placement and the exercise of bladder function before the removal of the catheter helps to deal with the issue.

Although there have been limited experiences of applying the La-TaTME to treat mid-low rectal cancer and the followup time is relatively short, La-TaTME provides a new surgical option for those low rectal cancer patients who are obese, have a narrow pelvis or undergone neoadjuvant radiochemotherapy. Based upon our research experiences, La-TaTME could be performed with safety and effectiveness if it is carefully conducted by experienced surgeons. However, multi-center randomized trials of La-TaTME, in comparison with traditional TME surgery, "are" still needed to further verify its oncological effects.

\section{Financial Support and Competing Interests}

The Authors declare that they have no competing interests.

\section{Acknowledgements}

This study was supported by a grant of the Clinical Research Launching Project of Beijing Friendship Hospital, Beijing, P.R. China (YYQDKT2016-5) and a grant of the Clinical Key Project of Peking University Third Hospital, Beijing, P.R. China (BYSY2016018).

\section{References}

1 Heald RJ, Husband EM and Ryall RD: The mesorectum in rectal cancer surgery - The clue to pelvic recurrence? Br J Surg 69(10): 613-616, 1982.

2 Bosch SL and Nagtegaal ID: The importance of the pathologist's role in assessment of the quality of the mesorectum. Curr Colorectal Cancer Rep 8(2): 90-98, 2012.

3 Tuech JJ, Karoui M, Lelong B, De Chaisemartin C, Bridoux V, Manceau G, Delpero JR, Hanoun L and Michot F: A step toward NOTES total mesorectal excision for rectal cancer: endoscopic transanal proctectomy. Ann Surg 261(2): 228-233, 2015.

4 Marks J, Mizrahi B, Dalane S, Nweze I and Marks G: Laparoscopic transanal abdominal transanal resection with sphincter preservation for rectal cancer in the distal $3 \mathrm{~cm}$ of the rectum after neoadjuvant therapy. Surg Endosc 24(11): 2700-2707, 2010.

5 van Gijn W, Marijnen CA, Nagtegaal ID, Kranenbarg EM, Putter H, Wiggers T, Rutten HJ, Påhlman L, Glimelius B and van de Velde CJ: Dutch Colorectal Cancer Group Preoperative radiotherapy combined with total mesorectal excision for resectable rectal cancer: 12-year follow-up of the multicentre, randomised controlled TME trial. Lancet Oncol 12(6): 575-582, 2011.

6 Kang SB, Park JW, Jeong SY, Nam BH, Choi HS, Kim DW, Lim SB, Lee TG, Kim DY, Kim JS, Chang HJ, Lee HS, Kim SY, Jung KH, Hong YS, Kim JH, Sohn DK, Kim DH and Oh JH: Open versus laparoscopic surgery for mid or low rectal cancer after neoadjuvant chemoradiotherapy (COREAN trial): Shortterm outcomes of an open-label randomised controlled trial. Lancet Oncol 11(7): 637-645, 2010.

7 van der Pas MH, Haglind E, Cuesta MA, Fürst A, Lacy AM, Hop WC and Bonjer HJ: COlorectal cancer Laparoscopic or Open Resection II (COLOR II) Study Group. Laparoscopic versus open surgery for rectal cancer (COLOR II): Short-term outcomes of a randomised, phase 3 trial. Lancet Oncol 14(3): 210-218, 2013.

8 Jeong SY, Park JW, Nam BH, Kim S, Kang SB, Lim SB, Choi HS, Kim DW, Chang HJ, Kim DY, Jung KH, Kim TY, Kang GH, Chie EK, Kim SY, Sohn DK, Kim DH, Kim JS, Lee HS, Kim JH and $\mathrm{Oh} \mathrm{JH}$ : Open versus laparoscopic surgery for mid-rectal or low-rectal cancer after neoadjuvant chemoradiotherapy (COREAN trial): Survival outcomes of an open-label, non-inferiority, randomised controlled trial. Lancet Oncol 15(7): 767-774, 2013.

9 Bonjer HJ, Deijen CL, Abis GA, Cuesta MA, van der Pas MH, de Lange-de Klerk ES, Lacy AM, Bemelman WA, Andersson J, Angenete E, Rosenberg J, Fuerst A and Haglind E: COLOR II Study Group. A randomized trial of laparoscopic versus open surgery for rectal cancer. N Engl J Med 372(14): 1324-1332, 2015. 
10 Fleshman J, Branda M, Sargent DJ, Boller AM, George V, Abbas M, Peters WR Jr., Maun D, Chang G, Herline A, Fichera A, Mutch M, Wexner S, Whiteford M, Marks J, Birnbaum E, Margolin D, Larson D, Marcello P, Posner M, Read T, Monson J, Wren SM, Pisters PW and Nelson H: Effect of laparoscopicassisted resection $v s$. open resection of stage II or III rectal cancer on pathologic outcomes: The ACOSOG Z6051 Randomized Clinical Trial. JAMA 314(13): 1346-1355, 2015.

11 Stevenson AR, Solomon MJ, Lumley JW, Hewett P, Clouston AD, Gebski VJ, Davies L, Wilson K, Hague W and Simes J: ALaCaRT Investigators. Effect of laparoscopic-assisted resection vs. open resection on pathological outcomes in rectal cancer: The ALaCaRT Randomized Clinical Trial. JAMA 314(13): 1356-1363, 2015.

12 Muratore A, Mellano A, Marsanic P and De Simone M: Transanal total mesorectal excision (taTME) for cancer located in the lower rectum: Short- and mid-term results. Eur J Surg Oncol 41(4): 478-483, 2015.

13 Hompes R, Arnold S and Warusavitarne J: Towards the safe introduction of transanal total mesorectal excision: The role of a clinical registry. Colorectal Dis 16(7): 498-501, 2014.

14 Battersby NJ, How P, Moran B, Stelzner S, West NP, Branagan G, Strassburg J, Quirke P, Tekkis P, Pedersen BG, Gudgeon M, Heald B and Brown G: MERCURY II Study Group. Prospective validation of a low rectal cancer magnetic resonance imaging staging system and development of a local recurrence risk stratification model: The MERCURY II Study. Ann Surg 263(4): 751-760, 2016.

15 Fernández-Hevia $\mathrm{M}$, Delgado $\mathrm{S}$, Castells A, Tasende $\mathrm{M}$, Momblan D, Díaz del Gobbo G, DeLacy B, Balust J and Lacy AM: Transanal total mesorectal excision in rectal cancer: Shortterm outcomes in comparison with laparoscopic surgery. Ann Surg 261(2): 221-227, 2015.
16 de Lacy AM, Rattner DW, Adelsdorfer C, Tasende MM, Fernández M, Delgado S, Sylla $\mathrm{P}$ and Martínez-Palli G: Transanal natural orifice transluminal endoscopic surgery (NOTES) rectal resection:“down-to-up"total mesorectal excision (TME)-Short-term outcomes in the first 20 cases. Surg Endosc 27(9): 3165-3172, 2013.

17 Nagtegaal ID, van de Velde CJ, van der Worp E, Kapiteijn E, Quirke P and van Krieken JH: Cooperative Clinical Investigators of the Dutch Colorectal Cancer Group. Macroscopic evaluation of rectal cancer resection specimen: Clinical significance of the pathologist in quality control. J Clin Oncol 20(7): 1729-1734, 2002.

18 Pulitanò C, Castillo F, Aldrighetti L, Bodingbauer M, Parks RW, Ferla G, Wigmore SJ and Garden OJ: What defines 'cure' after liver resection for colorectal metastases? Results after 10 years of follow-up. HBP 12(4): 244-249, 2010.

19 Rouanet P, Mourregot A, Azar CC, Carrere S, Gutowski M, Quenet F, Saint-Aubert B and Colombo PE: Transanal endoscopic proctectomy: An innovative procedure for difficult resection of rectal tumors in men with narrow pelvis. Dis Colon Rectum 56(4): 408-415, 2013. 